\title{
Antiferromagnetic noise correlations in optical lattices
}

\section{Citation}

Bruun, G. M., O. F. Syljuåsen, K. G. L. Pedersen, B. M. Andersen, E. Demler, and A. S. Sørensen. 2009. "Antiferromagnetic Noise Correlations in Optical Lattices." Physical Review A 80 (3) (September 28). doi:10.1103/physreva.80.033622.

\section{Published Version}

doi:10.1103/PhysRevA.80.033622

\section{Permanent link}

http://nrs.harvard.edu/urn-3:HUL.InstRepos:26370387

\section{Terms of Use}

This article was downloaded from Harvard University's DASH repository, and is made available under the terms and conditions applicable to Other Posted Material, as set forth at http:// nrs.harvard.edu/urn-3:HUL.InstRepos:dash.current.terms-of-use\#LAA

\section{Share Your Story}

The Harvard community has made this article openly available.

Please share how this access benefits you. Submit a story.

\section{Accessibility}




\title{
Antiferromagnetic noise correlations in optical lattices
}

\author{
G. M. Bruun, ${ }^{1,2}$ O. F. Syljuåsen, ${ }^{3}$ K. G. L. Pedersen, ${ }^{4}$ B. M. Andersen, ${ }^{4}$ E. Demler, ${ }^{5}$ and A. S. Sørensen ${ }^{4}$ \\ ${ }^{1}$ Niels Bohr International Academy, University of Copenhagen, DK-2100 Copenhagen $\varnothing$, Denmark \\ ${ }^{2}$ Mathematical Physics, Lund Institute of Technology, P.O. Box 118, SE-22100 Lund, Sweden \\ ${ }^{3}$ Department of Physics, University of Oslo, P.O. Box 1048, Blindern, N-0316 Oslo, Norway \\ ${ }^{4}$ Niels Bohr Institute, University of Copenhagen, DK-2100 Copenhagen $\varnothing$, Denmark \\ ${ }^{5}$ Department of Physics, Harvard University, Cambridge, Massachusetts 02138, USA
}

(Received 3 July 2009; published 28 September 2009)

\begin{abstract}
We analyze how noise correlations probed by time-of-flight experiments reveal antiferromagnetic (AF) correlations of fermionic atoms in two-dimensional and three-dimensional optical lattices. Combining analytical and quantum Monte Carlo calculations using experimentally realistic parameters, we show that AF correlations can be detected for temperatures above and below the critical temperature for AF ordering. It is demonstrated that spin-resolved noise correlations yield important information about the spin ordering. Finally, we show how to extract the spin correlation length and the related critical exponent of the AF transition from the noise.
\end{abstract}

DOI: $10.1103 /$ PhysRevA.80.033622

PACS number(s): 67.85.-d, 05.40.Ca, 75.40.Cx, 75.50.Ee

Atoms in optical lattices hold the potential to unravel the fundamental physics of phenomena related to quantum systems in periodic potentials including spin phases and high- $T_{c}$ superconductors [1]. One has already observed the Mott insulator transition for bosons [2], the emergence of superexchange interactions [3], the transition between metallic, band-insulator and Mott phases for fermions [4,5], and fermionic pairing [6]. A major challenge is how to detect the various phases predicted theoretically. These phases can be investigated via higher-order correlation functions which only show up as quantum noise in most experiments. Quantum spin noise spectroscopy $[7,8]$ and the measurement of correlations of the momentum distribution of the atoms after release from the lattice [time-of-flight (TOF) experiments] are two ways to probe these correlation functions [9]. TOF experiments have already been used to detect pairing correlations in a Fermi gas [10], bosonic bunching and fermionic antibunching of atoms in optical lattices [11], the Mottsuperfluid transition for bosons [12], and the effects of disorder in the Mott phase [13].

Here, we show how antiferromagnetic (AF) correlations of fermionic atoms in optical lattices give rise to distinct measurable signals in TOF experiments even above the critical temperature for magnetic ordering. While such correlations have been discussed before [9], we present the first quantitative calculation of the possible correlations over a large range of temperatures. These quantitative results may serve as a guideline to future experiments in this direction as well as an important tool for the interpretation of such experiments. Furthermore, spin-resolved experiments are demonstrated to yield additional information which can be used to identify the magnetic ordering and broken symmetry axis [14]. The main results are illustrated in Figs. 1 and 2 which show noise correlations in the momentum distributions after expansion as a function of temperature and momentum. We finally discuss how the spin correlation length and the related critical exponent $\nu$ can be extracted experimentally from the noise.

We consider a two-component Fermi gas in an optical lattice of size $N=N_{x} N_{y} N_{z}$. In the limit of strong repulsion the gas is in the Mott phase for low $T$ at half-filling and can be described by the Heisenberg model

$$
\hat{H}=J \sum_{\langle l, m\rangle}\left[\hat{s}_{l}^{x} \hat{s}_{m}^{x}+\hat{s}_{l}^{y} \hat{s}_{m}^{y}+(1+\Delta) \hat{s}_{l}^{z} \hat{s}_{m}^{z}\right] .
$$

Here $\hat{\mathbf{s}}_{l}$ is the spin-1/2 operator for atoms at site $\mathbf{r}_{l}$ and $\langle l, m\rangle$ denotes neighboring pairs [8]. The interaction is $J=4 t_{\uparrow} t_{\downarrow} / U$, with $U>0$ the on-site repulsion between atoms and $t_{\sigma}$ the spin-dependent tunneling matrix element. The anisotropy parameter is $\Delta=-\left(t_{\uparrow}-t_{\downarrow}\right)^{2} /\left(t_{\uparrow}^{2}+t_{\downarrow}^{2}\right)$. Below we consider both cubic [three dimensional (3D)] and square [two dimensional (2D)] lattices with lattice constant $a$ of unity. We do exclude any effects of a trapping potential.

A major experimental goal is to detect the onset of AF correlations with decreasing $T$. TOF experiments probe correlation functions of the form [11]

$$
C_{A B}\left(\mathbf{r}-\mathbf{r}^{\prime}\right)=\frac{\left\langle\hat{A}(\mathbf{r}) \hat{B}\left(\mathbf{r}^{\prime}\right)\right\rangle-\langle\hat{A}(\mathbf{r})\rangle\left\langle\hat{B}\left(\mathbf{r}^{\prime}\right)\right\rangle}{\langle\hat{A}(\mathbf{r})\rangle\left\langle\hat{B}\left(\mathbf{r}^{\prime}\right)\right\rangle},
$$

where $\hat{A}(\mathbf{r})$ and $\hat{B}(\mathbf{r})$ are atomic observables measured at $\mathbf{r}$ after expansion. In Refs. $[10,11]$, the atomic density correlations $C_{\mathrm{nn}}(\mathbf{d})$ were measured with $\hat{A}(\mathbf{r})=\hat{B}(\mathbf{r})=\Sigma_{\sigma} \hat{n}_{\sigma}(\mathbf{r})$ the

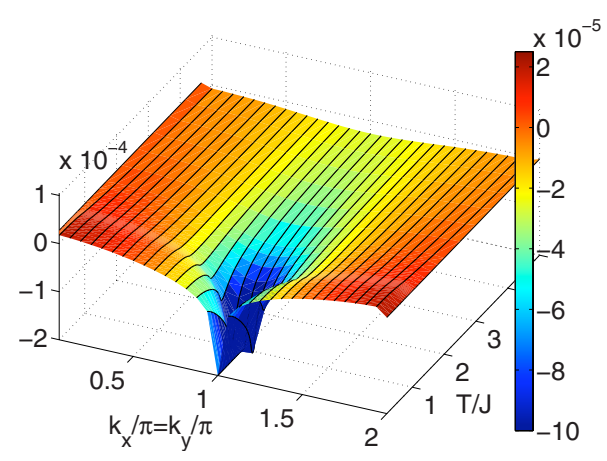

FIG. 1. (Color online) Plot of $C_{\mathrm{nn}}^{\exp }\left(\mathbf{k}_{\perp}\right)$ versus temperature $T$ and momentum along a diagonal cut $k=k_{x}=k_{y}$. 


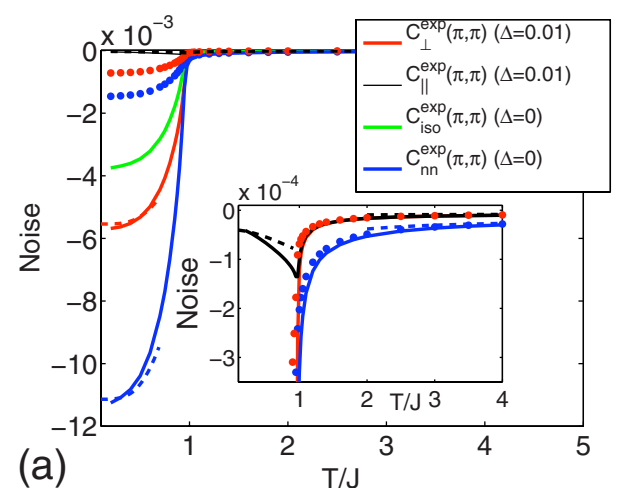

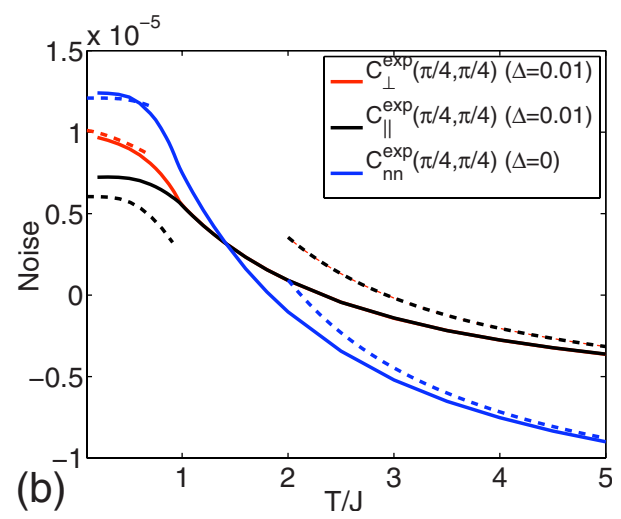

FIG. 2. (Color online) $C_{\perp}^{\exp }\left(\mathbf{k}_{\perp}\right) \quad\left[\right.$ red (gray)], $C_{\|}^{\exp }\left(\mathbf{k}_{\perp}\right)$ (black), $C_{\text {iso }}^{\exp }\left(\mathbf{k}_{\perp}\right) \quad$ [green (light gray)], and $C_{\mathrm{nn}}^{\exp }\left(\mathbf{k}_{\perp}\right)$ [blue (dark gray)] at $\mathbf{k}_{\perp}=(\pi, \pi)$ (a) and $\mathbf{k}_{\perp}$ $=(\pi / 4, \pi / 4) \quad$ (b). The dotted (solid) lines are with (without) Gaussian smearing $(\kappa=1 / 40)$. Dashed lines show the analytical results (7)-(9). Inset (a): same plot but zoomed in near $T_{N}$. density operator of the atoms. Here $\hat{n}_{\sigma}(\mathbf{r})=\hat{\psi}_{\sigma}^{\dagger}(\mathbf{r}) \hat{\psi}_{\sigma}(\mathbf{r})$ with $\hat{\psi}_{\sigma}(\mathbf{r})$ the field operator for atoms in spin state $\sigma$. We also consider the correlations between images of each of the spin components $C_{\|}(\mathbf{d})$ corresponding to $\hat{A}(\mathbf{r})=\hat{n}_{\uparrow}(\mathbf{r})$ and $\hat{B}(\mathbf{r})=\hat{n}_{\downarrow}(\mathbf{r})$. This may be achieved by, e.g., spatially separating the two atomic spin states using a Stern-Gerlach technique [15]. By applying a $\pi / 2$ pulse before the expansion one can also gain access to the spin noise perpendicular to the $z$ component $C_{\perp}(\mathbf{d})$ corresponding to $\hat{A}(\mathbf{r})=\exp \left(i \hat{s}^{y} \pi / 2\right) \hat{n}_{\uparrow}(\mathbf{r}) \exp \left(-i \hat{s}^{y} \pi / 2\right) \quad$ and $\hat{B}(\mathbf{r})$ $=\exp \left(i \hat{S}^{y} \pi / 2\right) \hat{n}_{\downarrow}(\mathbf{r}) \exp \left(-i \hat{s}^{y} \pi / 2\right)$.

After normal ordering and expansion of the field operators in the lowest band Wannier states, we obtain

$$
\begin{gathered}
C_{\mathrm{nn}}(\mathbf{k})=\frac{1}{N}-\frac{1}{2} \delta_{\mathbf{k}, \mathbf{K}}-2\left\langle\hat{\mathbf{s}}_{\mathbf{k}} \cdot \hat{\mathbf{s}}_{-\mathbf{k}}\right\rangle, \\
C_{\|}(\mathbf{k})=\frac{1}{2 N}-\left\langle\hat{s}_{0}^{z} \hat{s}_{0}^{z}\right\rangle-\left\langle\hat{s}_{\mathbf{k}}^{x} \hat{s}_{-\mathbf{k}}^{x}\right\rangle-\left\langle\hat{s}_{\mathbf{k}}^{y} \hat{s}_{-\mathbf{k}}^{y}\right\rangle, \\
C_{\perp}(\mathbf{k})=\frac{1}{2 N}-\left\langle\hat{s}_{0}^{x} \hat{s}_{0}^{x}\right\rangle-\left\langle\hat{s}_{\mathbf{k}}^{y} \hat{s}_{-\mathbf{k}}^{y}\right\rangle-\left\langle\hat{s}_{\mathbf{k}}^{z} \hat{s}_{-\mathbf{k}}^{z}\right\rangle,
\end{gathered}
$$

where $\mathbf{K}$ is a reciprocal lattice vector, and $\hat{\mathbf{s}}_{\mathbf{k}}=N^{-1} \sum_{l} \hat{\mathbf{s}}_{l} e^{-i \mathbf{k} \cdot \mathbf{r}_{l}}$. We assume free expansion of the atoms for a duration $t$, neglect autocorrelation terms $\propto \delta(\mathbf{d})$ in Eqs. (3)-(5), and express $C$ in terms of the momentum $\mathbf{k}=m \mathbf{d} / t(\hbar=1$ and momenta are measured in units of $1 / a$ throughout). These correlation functions have contributions with different scalings. For 3D systems, the spins order below the Néel temperature $T_{N}$. Assuming the broken symmetry axis along the $z$ axis, $C_{\mathrm{nn}}$ and $C_{\perp}$ have a contribution from $\left\langle\hat{s}_{\mathbf{k}}^{z} \hat{s}_{-\mathbf{k}}^{z}\right\rangle \sim O(1)$ at $\mathbf{k}=(\pi, \pi, \pi)$ for $T<T_{N}$. At other momenta or for $T>T_{N}$ the correlation functions scale as $1 / N$. We will perform calculations where the total spin $\propto \hat{s}_{0}^{z}$ of the lattice is allowed to fluctuate, which is different from the experimental situation where the number of particles in each spin state is fixed. This may give rise to different momentum independent terms in Eqs. (3)-(5) for the experiment, but the $k$-dependent part should be accurately captured by our calculations.

Experiments take 2D column density images of the expanding cloud corresponding to integrating over $z$ and $z^{\prime}$ in both the numerator and denominator in Eq. (2). The cameras also introduce a smoothening in the $x y$ plane, which can be modeled by convolution with a Gaussian [11]. In total, the experimental procedure corresponds to measuring the averaged correlation function

$$
C^{\exp }\left(\mathbf{k}_{\perp}\right)=\frac{1}{4 \pi N \kappa^{2}} \sum_{\mathbf{k}^{\prime}} e^{-1 / 4 \kappa^{2} 1 /(2 \pi)^{2}\left(\mathbf{k}_{\perp}^{\prime}-\mathbf{k}_{\perp}\right)^{2}} C\left(\mathbf{k}^{\prime}\right),
$$

where $\mathbf{k}_{\perp}=\left(k_{x}, k_{y}\right)$ and $\kappa=w / l$ with $l=2 \pi t / m a$ (keeping $a$ for clarity) and $w$ a width depending on the pixel resolution of the charge-coupled device camera. The averaging in the $z$-direction reduces the contributions to $C_{\mathrm{nn}}$ and $C_{\perp}$ at $\mathbf{k}=(\pi, \pi, \pi)$ from $O(1)$ for $T<T_{N}$ to $1 / N_{z}$ and the Gaussian smoothening further reduces it to $O\left(1 / \kappa^{2} N\right)$. This reduction happens because the correlations are restricted to a single point $\mathbf{k}=(\pi, \pi, \pi)$ and we are averaging over $N_{z} \cdot N_{x} N_{y} \kappa^{2}$ points. Away from $\mathbf{k}_{\perp}=(\pi, \pi)$ or for $T>T_{N}$ the correlations have a wider distribution and they are less affected by the averaging.

We plot in Fig. $1 C_{\mathrm{nn}}^{\exp }(k, k)$ as a function of $k=k_{x}=k_{y}$ and $T$ for a 3D lattice of size $N=32^{3}$. Quantum Monte Carlo (QMC) simulations using the stochastic series expansion method [16] with directed-loop updates [17] were used to calculate the correlation functions $\left\langle\hat{\mathbf{s}}_{\mathbf{k}} \cdot \hat{\mathbf{s}}_{-\mathbf{k}}\right\rangle$. This method is very efficient for Heisenberg models and gives accurate results for a wide range of $T$ for large systems. For the isotropic Heisenberg model, the QMC calculations yield $T_{N} \simeq 0.945 \mathrm{~J}$, in agreement with Ref. [18] $C_{\mathrm{nn}}^{\exp }$, was then calculated from Eq. (3) with the $k_{z}$ average in Eq. (6) included to simulate the experimentally relevant situation [11]. The main feature of the plot is the dip at $k=\pi$ coming from AF ordering. For $T<T_{N}$, this gives rise to a large Bragg dip at $k_{x}=k_{y}=\pi[9,19]$. We see that the Bragg dip remains also above $T_{N}$ but has a larger width due to AF correlations without long range order. The width of the dip decreases with decreasing $T-T_{N}$ reflecting that the spin correlation length $\xi$ increases as $\xi \sim 1 /\left|T-T_{N}\right|^{\nu}$ for $T \rightarrow T_{N}^{+}$. We show below how the noise can be used to extract the critical exponent $\nu$. At high $T$ the AF correlations lead to singlet formation of neighboring spins. This gives rise to a precursor of the Bragg peak at $k_{x}=k_{y}=\pi$ and an equal signal of opposite sign at $\mathbf{k}_{\perp}=0$ [see Eq. (7)]. These momentum correlations can be understood by noting that two fermions in a singlet are more (less) likely to have the same (opposite) momentum due to the Pauli exclusion principle. The uniform spin noise case $k=0$ was also considered in [8]. 

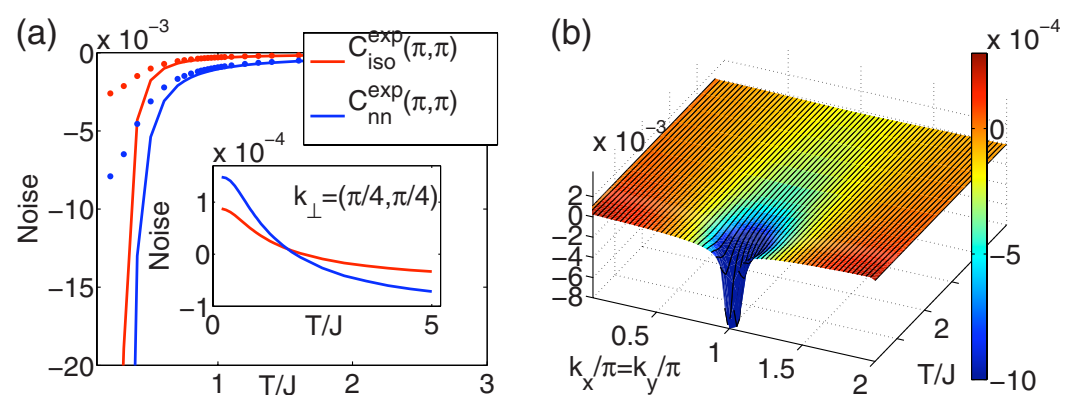

FIG. 3. (Color online) (a) Correlation functions $C_{\text {iso }}^{\exp }\left(\mathbf{k}_{\perp}\right)$ [red (gray)] and $C_{\mathrm{nn}}^{\exp }\left(\mathbf{k}_{\perp}\right)$ [blue (dark gray)] at $\mathbf{k}_{\perp}=(\pi, \pi)$ for a $2 \mathrm{D}$ lattice. The curves are obtained from QMC simulations on $64 \times 64$ lattices. The dotted (solid) lines are with (without) Gaussian smearing using $\kappa=1 / 40$. Inset: same as solid lines in main panel except at $\mathbf{k}_{\perp}=(\pi / 4, \pi / 4)$. (b) Same as Fig. 1 but for a $2 \mathrm{D}$ system without the $k_{z}$ summation.
In the high- $T$ limit $J / T=1 / \widetilde{T} \ll 1\left(k_{B}=1\right)$, controlled analytical results for the correlation functions (3)-(5) may be obtained by expanding in $\tilde{T}^{-1}$. We obtain

$$
\begin{gathered}
C_{\mathrm{nn}}(\mathbf{k})=-\frac{1}{2} \delta_{\mathbf{k}, \mathbf{k}}-\frac{1}{2 N}+\frac{3 Z \gamma_{\mathbf{k}}}{8 N \tilde{T}}+O\left(\tilde{T}^{-2}\right), \\
C_{\|}(\mathbf{k})=C_{\perp}(\mathbf{k})=-\frac{1}{4 N}+\frac{Z}{8 N \tilde{T}}\left(\frac{1}{2}+\gamma_{\mathbf{k}}\right)+O\left(\tilde{T}^{-2}\right),
\end{gathered}
$$

with $Z=4(6)$ for 2D (3D) lattices. Here, $\gamma_{\mathbf{k}}$ $=Z^{-1} \sum_{\mathbf{a}} 2 \cos (\mathbf{k} \cdot \mathbf{a})$, where $\Sigma_{\mathbf{a}}$ sums over the unit vectors spanning the lattice and we have taken $\Delta=0$ for simplicity. The average of Eqs. (7) and (8) over $z$ can be obtained by simply omitting the $z$ direction in the sum.

To obtain analytic expressions for low $T$, we perform a spin-wave calculation, which should be fairly accurate for a $3 \mathrm{D}$ system at $T \ll T_{N}$. This yields after some algebra

$$
C_{\|}(\mathbf{k})=\frac{1}{2 N}-\left\langle\hat{s}_{0}^{z} \hat{s}_{0}^{z}\right\rangle-\frac{1}{2 N}\left(1+2 f_{\mathbf{k}}\right) e^{-2 \Theta_{\mathbf{k}}},
$$

where tanh $2 \Theta_{\mathbf{k}}=\gamma_{\mathbf{k}} /(1+\Delta)$ and $f_{\mathbf{k}}=\left[\exp \omega_{\mathbf{k}} / T-1\right]^{-1}$ is the Bose distribution function for the spin waves with energy $\omega_{\mathbf{k}}=3 J \sqrt{(1+\Delta)^{2}-\gamma_{\mathbf{k}}^{2}}$. We also have $\left\langle\hat{s}_{0}^{z} \hat{s}_{0}^{z}\right\rangle$ $=\sum_{\mathbf{k}}^{\prime} \sinh ^{-2}\left(\beta \omega_{\mathbf{k}} / 2\right) / 2 N^{2}$ where the sum extends over the AF reduced Brillouin zone [8]. A similar but somewhat more lengthy expression can be derived for $C_{\perp}(\mathbf{k})$.

We plot in Fig. $2 C_{\mathrm{nn}}^{\exp }\left(\mathbf{k}_{\perp}\right), C_{\|}^{\exp }\left(\mathbf{k}_{\perp}\right)$, and $C_{\perp}^{\exp }\left(\mathbf{k}_{\perp}\right)$ as a function of $T$ for $\mathbf{k}_{\perp}=(\pi, \pi)$ (a) and $\mathbf{k}_{\perp}=(\pi / 4, \pi / 4)$ (b) for the same parameters as above. The solid curves include the average over $k_{z}$ only and the dotted curves include a Gaussian smoothing in the $\left(k_{x}, k_{y}\right)$ plane as well. The values for $T \rightarrow 0$ with Gaussian smearing are consistent with the results of Ref. [11] given the different system size and slightly different measured quantities. For simplicity we have excluded the Gaussian averaging for $\mathbf{k}_{\perp}=(\pi / 4, \pi / 4)$ since it [contrary to the $\mathbf{k}_{\perp}=(\pi, \pi)$ case] leads to only negligible changes. We trigger the AF ordering along the $z$ direction for $T<T_{N}$ by including a small anisotropy $\Delta=0.01$ for $C_{\|}^{\exp }$ and $C_{\perp}^{\exp }$.

Figure 2(a) shows the Bragg dip for $T<T_{N}$ coming from $\left\langle\hat{s}_{\mathbf{k}}^{z} s_{-\mathbf{k}}^{z}\right\rangle \approx\left|\left\langle s_{i}^{z}\right\rangle\right|^{2}$ for $\mathbf{k}=(\pi, \pi, \pi)$ with $\left|\left\langle s_{i}^{z}\right\rangle\right|>0$ the staggered magnetization. Extrapolating to $T=0$ we have $\left|\left\langle s_{i}^{z}\right\rangle\right| \simeq 0.43 \pm 0.01$, a value reduced from $1 / 2$ by quantum fluctuations. On the other hand the spin noise parallel to the broken symmetry axis $C_{\|}$is reduced with the onset of magnetic ordering for $T<T_{N}$ as can be seen from the inset in Fig. 2(a). (The minimal value of $C_{\|}$at $T_{N}$ is dependent on the anisotropy $\Delta$.) Note that even though the density correlations are larger and hence more easily measurable, the spinresolved measurements are crucial in verifying, e.g., whether the correlation dip is indeed due to magnetic ordering and not caused, for example, by formation of a period-doubled charge-density wave. The difference between $C_{\|}$and $C_{\perp}$ for $\Delta>0$ can furthermore be used to identify the broken symmetry axis. In the isotropic case, there is no broken symmetry axis and $C_{\|}^{\exp }=C_{\perp}^{\exp }=C_{\text {iso }}^{\exp }$ for $\Delta \rightarrow 0$, as can be seen from the green (light gray) curve in Fig. 2(a).

The calculated correlations are rather small even for the quantities including the macroscopic contribution $\left\langle\hat{s}_{\mathbf{k}}^{z} s_{-\mathbf{k}}^{z}\right\rangle \approx\left|\left\langle s_{i}^{z}\right\rangle\right|^{2}$ at $\mathbf{k}=(\pi, \pi, \pi)$, e.g., for low temperatures $T \leqq J / 2 C_{\mathrm{nn}}^{\exp } \approx 10^{-2}\left(10^{-3}\right)$ without (with) Gaussian smoothening and scales like $1 / N_{z}(1 / N)$. This is, however, still significantly larger than the correlations of order $C_{\mathrm{nn}} \sim 10^{-4}$, which were recently measured with slightly bigger system sizes [11]. The correlations are also close to the measured experimental values even for temperatures above $T_{N}$, i.e., for $T=2 J$ we have $C_{\mathrm{nn}}^{\exp } \approx 6 \times 10^{-5}$ (scaling as $\left.1 / N\right)$, which is comparable to the recent experiments [11]. The measurement of noise correlations can thus be used to show the onset of AF order even above the critical temperature. Furthermore, for current experiments the spin temperature is uncertain because there are no sensitive probes. If the noise correlations are measured, the detailed theoretical curves presented here would provide a means of assessing the spin temperature in the experiments.

The density and spin noise at $\mathbf{k}_{\perp}=(\pi / 4, \pi / 4)$ depicted in Fig. 2(b) exhibit a different behavior from that at $\mathbf{k}_{\perp}=(\pi, \pi)$ : it now decreases in numerical value for high $T$ and even changes sign above $T_{N}$. This unusual behavior is a geometric effect of the lattice. Note that since the noise scales as $1 / N$ away from the Bragg point, a measurement requires higher experimental resolution than what is presently available or more sensitive detection methods such as spin noise spectroscopy [8].

Atomic gases are well suited to study fundamental problems in 2D physics as the observation of the BerezinskiiKosterlitz-Thouless transition illustrates [20]. Recently, single layer 2D atomic gases have been produced which avoids the averaging over $z$ discussed above [21]. We now study the Mott phase at half-filling for a 2D square lattice. In 2D there is no ordered phase for $T>0$ due to fluctuations, but there are still significant AF correlations [22]. This is illustrated in Fig. 3(a), which shows $C_{\text {iso }}^{\exp }\left(\mathbf{k}_{\perp}\right)$ and $C_{\mathrm{nn}}^{\exp }\left(\mathbf{k}_{\perp}\right)$ as a function of $T$. The AF correlations give rise to a large dip for $\mathbf{k}_{\perp}=(\pi, \pi)$ both in the density and spin noise which is a precursor of the AF ordered state at $T=0$. Since there is 

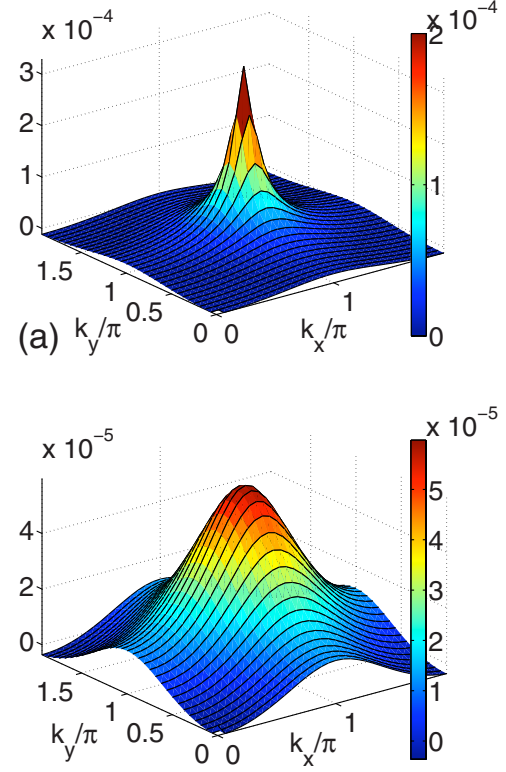

(c)
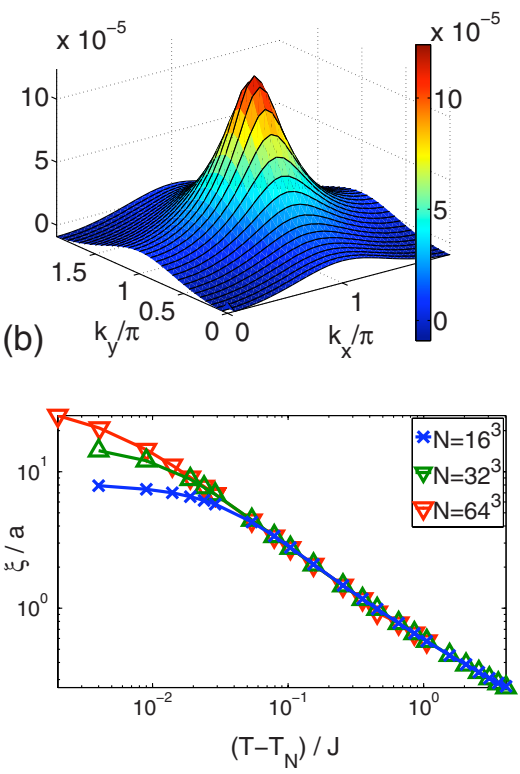

(d)

no averaging over the $z$ direction, the correlations are much stronger than for a $3 \mathrm{D}$ system above $T_{N}$. Figure 3 illustrates that the noise exhibits the same nontrivial features as a function of $k$ and $T$ as the 3D case.

Critical exponents characterizing continuous phase transitions are often difficult to measure. We now demonstrate how noise measurements can be used to obtain the critical exponent $\nu$. The correlation length $\xi$ can be extracted from the width of the AF dip in the $3 \mathrm{D}$ resolved function $C_{\mathrm{nn}}(\mathbf{k})$ at $\mathbf{k}=(\pi, \pi, \pi)$. We find that this yields a critical exponent $\nu \approx 0.70$ as expected for a 3D Heisenberg model [23]. Importantly, $\nu$ can also be extracted from the experimentally relevant $k_{z}$-summed correlation function $C_{\mathrm{nn}}^{\text {exp }}$. Figures 4(a)-4(c) show $C_{\mathrm{nn}}^{\exp }\left(\mathbf{k}_{\perp}\right)$ at three fixed temperatures above $T_{N}$. To obtain $\nu$, we fit $C_{\mathrm{nn}}^{\exp }\left(\mathbf{k}_{\perp}\right)$ to a summed lattice propagator of the form $\Sigma_{k_{z}}\left\{2\left[3-\sum_{\alpha=x, y, z} \cos \left(k_{\alpha}-\pi\right)\right] \xi^{2}+1\right\}^{-1}$ with $k_{z}=2 \pi n_{z} / N_{z}$. Figure $4(d)$ shows the extracted correlation length $\xi$ for various system sizes. One clearly sees the finite-size effects setting in at decreasing $T-T_{N}$. The extracted power law yields $\nu \approx 0.70$. To obtain a robust value for $\nu$ one needs to probe the noise for temperatures where it is somewhat smaller than what has been measured to date. This would however enable one to probe the critical properties of the AF transition.

In summary, we performed analytic and numerical calculations modeling TOF experiments for repulsive fermionic atoms in optical lattices using experimentally realistic parameters. This demonstrated that such experiments are well suited to detect AF correlations both below and above the critical temperature. Spin-resolved measurements were shown to yield valuable additional information and we finally discussed how to extract the critical exponent governing the correlation length close to the AF transition from the noise.

\section{ACKNOWLEDGMENTS}

We thank H. Moritz for the suggestion to look at TOF experiments. The QMC simulations were carried out using resources provided by the NOTUR project of the Norwegian Research Council. B.M.A. acknowledges support from the Villum Kann Rasmussen foundation and E.D. from NSF under Grant No. DMR-0705472, CUA, DARPA, and MURI.
[1] W. Hofstetter, J. I. Cirac, P. Zoller, E. Demler, and M. D. Lukin, Phys. Rev. Lett. 89, 220407 (2002); L.-M. Duan, E. Demler, and M. D. Lukin, ibid. 91, 090402 (2003).

[2] M. Greiner et al., Nature (London) 415, 39 (2002).

[3] S. Trotzky et al., Science 319, 295 (2008).

[4] R. Jördens et al., Nature (London) 455, 204 (2008).

[5] U. Schneider et al., Science 322, 1520 (2008).

[6] J. K. Chin et al., Nature (London) 443, 961 (2006).

[7] K. Eckert et al., Nat. Phys. 4, 50 (2007).

[8] G. M. Bruun, B. M. Andersen, E. Demler, and A. S. Sorensen, Phys. Rev. Lett. 102, 030401 (2009).

[9] E. Altman, E. Demler, and M. D. Lukin, Phys. Rev. A 70,
013603 (2004).

[10] M. Greiner, C. A. Regal, J. T. Stewart, and D. S. Jin, Phys. Rev. Lett. 94, 110401 (2005).

[11] S. Fölling et al., Nature (London) 434, 481 (2005); T. Rom et al., ibid. 444, 733 (2006).

[12] I. B. Spielman, W. D. Phillips, and J. V. Porto, Phys. Rev. Lett. 98, 080404 (2007).

[13] V. Guarrera, N. Fabbri, L. Fallani, C. Fort, K. M. R. van der Stam, and M. Inguscio, Phys. Rev. Lett. 100, 250403 (2008).

[14] T. Gottwald and P. G. J. van Dongen, Phys. Rev. A 80, 033603 (2009).

[15] T. Esslinger (private communication). 
[16] A. W. Sandvik and J. Kurkijärvi, Phys. Rev. B 43, 5950 (1991).

[17] O. F. Syljuåsen and A. W. Sandvik, Phys. Rev. E 66, 046701 (2002).

[18] A. W. Sandvik, Phys. Rev. Lett. 80, 5196 (1998).

[19] B. M. Andersen and G. M. Bruun, Phys. Rev. A 76, 041602(R) (2007).

[20] Z. Hadzibabic et al., Nature (London) 441, 1118 (2006); V. Schweikhard, S. Tung, and E. A. Cornell, Phys. Rev. Lett. 99,
030401 (2007); P. Cladé, C. Ryu, A. Ramanathan, K. Helmerson, and W. D. Phillips, Phys. Rev. Lett. 102, 170401 (2009).

[21] N. Gemelke, X. Zhang, C. Hung, and C. Chin, Nature 460, 995 (2009); J. I. Gillen, W. S. Bakr, A. Peng, P. Unterwaditzer, S. Fölling, and M. Greiner, Phys. Rev. A 80, 021602 (2009).

[22] A. Auerbach, Interacting Electrons and Quantum Magnetism (Springer, New York, 1998).

[23] A. Pelissetto and E. Vicari, Phys. Rep. 368, 549 (2002). 